\title{
Biodegradable andrographolide-eluting nanofibrous membranes for the treatment of cervical cancer
}

This article was published in the following Dove Press journal: International Journal of Nanomedicine

\author{
Yi-Pin Chen ${ }^{1,2}$ \\ Yen-Wei Liu $^{3}$ \\ Demei Lee ${ }^{3}$ \\ Jiantai Timothy Qiu ${ }^{4,5}$ \\ Tzung-Yan Lee Le,7 $^{6}$ \\ Shih-Jung $\mathrm{Liu}^{3,8}$ \\ 'Graduate Institute of Clinical \\ Medical Sciences, College of Medicine, \\ Chang Gung University, Tao-Yuan, \\ Taiwan; ${ }^{2}$ Department of Obstetrics \\ and Gynecology, Keelung Chang \\ Gung Memorial Hospital, Keelung, \\ Taiwan; ${ }^{3}$ Department of Mechanical \\ Engineering, Chang Gung University, \\ Tao-Yuan, Taiwan; ${ }^{4}$ Department of \\ Obstetrics and Gynecology, Linkou \\ Chang Gung Memorial Hospital, \\ Tao-Yuan, Taiwan; ${ }^{5}$ Department of \\ Biomedical Sciences, College of \\ Medicine, Chang Gung University, \\ Tao-Yuan, Taiwan; ${ }^{6} \mathrm{G}$ raduate Institute \\ of Traditional Chinese Medicine, \\ School of Chinese Medicine, College \\ of Medicine, Chang Gung University, \\ Tao-Yuan, Taiwan; ${ }^{7}$ Department of \\ Traditional Chinese Medicine, Keelung \\ Chang Gung Memorial Hospital, \\ Keelung, Taiwan; ${ }^{8}$ Department of \\ Orthopedic Surgery, Linkou Chang \\ Gung Memorial Hospital, Tao-Yuan, \\ Taiwan
}

Background: In this study, we developed biodegradable andrographolide (AG)-eluting nanofibrous mats and evaluated their efficacy in treating cervical cancer.

Materials and methods: Membranes of two different poly[(D,L)-lactide-co-glycolide] (PLGA)to-AG ratios (6:1 and 3:1) were prepared via electrospinning technology. The liberation behavior of AG was evaluated. A cervical cancer model with C57BL/6J mice was created and employed for an in vivo efficacy assessment of the drug-eluting nanofibers. Twelve mice with cervical cancer were stochastically divided into three different groups (four animals per group): group A received no treatment as the control, group B was treated with pure PLGA mats, and group C was treated with AG-loaded nanofibrous membranes. The changes in tumor sizes were recorded.

Results: All membranes eluted high concentrations of AG at the target area for three weeks, while the systemic drug concentration in the blood remained low. Histological analysis showed no obvious tissue inflammation. Compared with the mice in groups A and B, the tumor size of the mice in group $\mathrm{C}$ decreased with time until day 25 , when the daily drug concentration reduced to $3 \mu \mathrm{g} / \mathrm{mL}$.

Conclusion: Biodegradable nanofibers with a sustainable release of AG exhibit adequate efficacy and durability for the treatment of mice with cervical cancer.

Keywords: andrographolide, cervical cancer, biodegradable nanofiber, sustainable release

\section{Introduction}

Cervical carcinoma is a highly potent gynecological malignancy and comprises $\sim 12 \%$ of all carcinomas in women worldwide. ${ }^{1}$ It originates in the cervix owing to the abnormal proliferation of cells, which are then capable of invading or migrating to other organs of the body. There are usually no observable symptoms in its early stages. Abnormal vaginal bleeding, pelvic pain, or pain during sexual intercourse may occur in its advanced stages. While bleeding after sex may commonly occur, it may also represent the occurrence of cervical cancer. ${ }^{2}$ Over $99 \%$ of cervical carcinoma cases are carriers of human papilloma viruses (HPVs), wherein HPV is a primary risk factor for cervical carcinoma. ${ }^{3} \mathrm{HPV}$ infection appears to be involved in the development of $>90 \%$ of cervical cancer cases. Nevertheless, most people who have had HPV infections do not develop cervical cancer. Other risk factors include smoking, birth control pills, and becoming sexually active at a young age. Cervical cancer generally evolves from precancerous changes over the span of 10-20 years. Approximately 69\% of cervical cancer instances are squamous cell carcinomas and $25 \%$ are adenocarcinoma, along with a few other forms. Cervical biopsy and medical imaging are typically used to diagnose these cancers.
Biomaterials Lab, Mechanical Engineering, Chang Gung University, 259, Wen-Hwa Ist Road, Kwei-Shan, Tao-Yuan 333, Taiwan

Tel +88632118166

Fax +88632118558

Email profsjliu5347@gmail.com 
Despite the fact that prophylactic HPV vaccines have been reported to prevent up to $90 \%$ of cervical carcinomas, having regular Pap smears, few or no sexual partners, and the use of condoms are still generally recommended. ${ }^{4}$ Cervical intraepithelial neoplasm (CIN) - a cervical pre-cancer lesion - can also be diagnosed by Pap smear or cervical biopsy. A combined therapeutic regime consisting of surgery, chemotherapy, and radiotherapy may be employed to treat cervical cancer. ${ }^{5}$ Despite these treatments, many cervical cancer patients die every year. The development of new therapeutic methods to effectively manage this dreadful disease in women is thus needed. ${ }^{6}$

Thus far, researchers have developed nanofibrous mats loaded with cisplatin and curcumin, ${ }^{7}$ dichloroacetate (DCA) and oxaliplatin, ${ }^{8}$ and $\mathrm{Pt}(\mathrm{IV})$ prodrug-backboned micelle and DCA-loaded nanofibers ${ }^{9}$ to reduce tumor recurrence for cervical cancer treatment. The experimental results have suggested that the implanted devices exhibit enhanced anticancer efficacy with lower systemic toxicity against advanced cervical cancer in vivo.

In this work, we developed biodegradable andrographolide (AG)-eluting nanofibers as an anticancer film that, when applied topically, provided the sustained release of anticancer pharmaceuticals at a target area. We fabricated biodegradable AG-loaded nanofibrous membranes to inhibit the progression of cervical cancer. AG is a labdane diterpenoid (Figure 1) that has been isolated from the stem and leaves of Andrographis paniculata, and has been traditionally used to treat infections and certain diseases. Recent studies have demonstrated the anticancer characteristics of $\mathrm{AG}$ and its derivatives, ${ }^{10-15}$ which are promising agents for the suppression of tumor metastasis. Furthermore, compared with most anticancer compounds, AG attenuates inflammation by the inhibition of nuclear factor kappa B activation through the covalent modification of reduced cysteine 62 of p50, demonstrating a unique pharmacological mechanism of protective anti-inflammatory actions. ${ }^{16}$ To fabricate the biodegradable

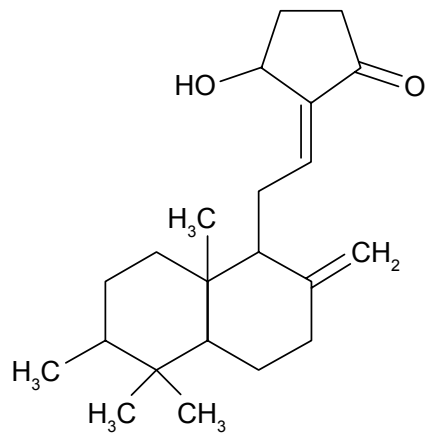

Figure I Chemical structure of andrographolide. nanofibers, $\mathrm{AG}$ and poly[(D,L)-lactide-co-glycolide] (PLGA) were mixed with hexafluoro-2-propanol (HFIP) and electrospun into nanofibrous mats. The fabricated nanofibers were then characterized. The in vitro and in vivo liberation behaviors of AG from the nanofibers were explored. The efficacy of the released AG against cervical tumors in mice was investigated. Histological analyses were also carried out.

\section{Materials and methods Manufacture of AG-eluting nanofibrous membranes}

The materials utilized included PLGA (LA:GA =50:50) polymer, with a molecular weight of $33 \mathrm{kDa}$. AG was used as the pharmaceutical drug. Both were acquired from SigmaAldrich (St Louis, MO, USA).

The nanofibers with two PLGA-to-AG ratios, namely 6:1 and 3:1, were produced utilizing an electrospinning setup. ${ }^{17}$ To fabricate the AG-embedded nanofibers with a 6:1 polymer-to-drug ratio, PLGA/AG (720:120 mg) were first mixed with $3 \mathrm{~mL}$ of HFIP (Sigma-Aldrich). The mixture was transported and spun by a syringe pump at a flow rate of $0.025 \mathrm{~mL} / \mathrm{min}$ into a non-woven form nanofibrous membrane onto a collection plate at room temperature. The voltage was set to $17 \mathrm{kV}$, while the travel distance from the needle to the collection plate was $9 \mathrm{~cm}$. The manufacture of nanofibers with a 3:1 polymer-to-drug ratio consisted of the same electrospinning process, except that the polymer-to-AG ratio utilized was 630:210 mg.

\section{Evaluation of spun nanofibrous membranes}

Spun nanofibers were assessed utilizing a scanning electron microscope (SEM) (JSM-7500F; Joel, Tokyo, Japan). Fifty arbitrarily chosen fibers from each SEM image were employed to calculate the fiber diameter distribution.

A contact angle measurement device (First Ten Angstroms, Portsmouth, VA, USA) ( $\mathrm{N}=5$ ) was used to measure the water contact angles of the electrospun membranes. Meanwhile, a Lloyd tensiometer (AMETEK, Berwyn, PA, USA) was employed to measure the tensile properties of the nanofibrous membranes.

A Fourier Transform Infrared (FTIR) spectrometer (Model Nicolet iS5 spectrometer; Thermo Scientific, Waltham, MA, USA) was utilized to investigate the spectra of electrospun AG-eluting nanofibers. Specimens were compressed as $\mathrm{KBr}$ discs, and spectra were monitored in the absorption modes at resolutions of $4 \mathrm{~cm}^{-1}$ and 32 scans over a $400-4,000 \mathrm{~cm}^{-1}$ range. 

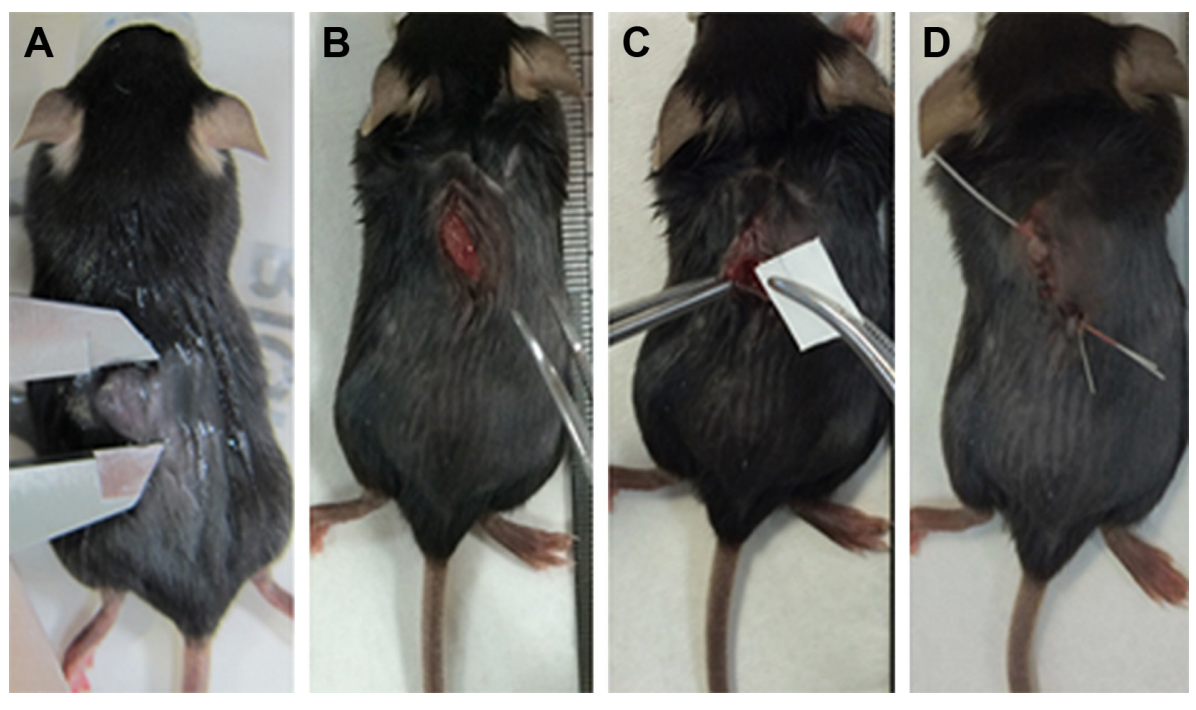

Figure 2 Experimental procedure of the deployment of the andrographolide-eluting membranes.

Notes: (A) A rat bearing the tumor. (B) An incision was made. (C) Implantation of nanofibrous membrane. (D) Wound was closed by sutures.

\section{Assessment of in vitro drug release}

The liberation behavior of AG from the drug-embedded nanofibrous membranes with various PLGA-to-drug ratios (6:1 and 3:1) was resolved utilizing the in vitro elution method. Specimens with a size of $10 \times 10 \mathrm{~mm}$ and a weight of $5 \mathrm{mg}$ were placed in $1 \mathrm{~mL}$ of buffered solution. The eluent was gathered and analyzed after being placed in an isothermal oven at $37^{\circ} \mathrm{C}$ for 24 hours. The phosphate buffer was substituted every 24 hours and the procedure was repeated for 40 days.

The AG levels were evaluated utilizing a highperformance liquid chromatography (HPLC) assay on a Hitachi L-2200R system (Hitachi, Ltd., Tokyo, Japan) using a Discovery ${ }^{\circledR} 25 \mathrm{~cm} \times 4.6 \mathrm{~mm}, 5 \mu \mathrm{m}$ HPLC column (Sigma-Aldrich). The mobile phase for the analysis included methanol (20/80; Sigma-Aldrich) and distilled water at a ratio of $62: 38[\mathrm{v} / \mathrm{v}]$. The absorbency was assessed using an ultraviolet light detector (L-2400R; Hitachi, Ltd.) at a wavelength of $250 \mathrm{~nm}$. The volumetric flow rate was $1.0 \mathrm{~mL} / \mathrm{min}$, while the retention time was 10 minutes.

\section{In vivo animal model assessment}

All animal-related procedures received institutional approval from Chang Gung University, and all of the animals were cared for under the supervision of a licensed veterinarian in a manner that was consistent with the regulations of the Department of Health and Welfare, Taiwan. Female C57BL/6J (B6) mice were acquired from the National Laboratory Animal Center (Taipei, Taiwan) and kept at the Animal Center of Chang Gung University. The animalrelated procedures acquired institutional approval. All of the animals employed were 6-8 weeks old.
E7-expressing tumorigenic cell line, HPV + TC-1, was obtained from Dr T.C. Wu at Johns Hopkins University in the USA. Mice were subcutaneously injected in the back region with $2 \times 10^{5} \mathrm{HPV}+\mathrm{TC}-1$ tumor cells/per mouse on day $0 .{ }^{18}$ The use of the cell line was approved by the institutional review board.

Mice were anesthetized via breathe-in of isoflurane utilizing an atomizer in an anesthesia box $(40 \times 20 \times 28 \mathrm{~cm})$. After shaving, an incision was made on the backs of the mice to expose the tumors. The mice were operated under anesthesia that was maintained by breath-in of isoflurane via a mask. All mice were divided into three groups with four mice in each group. The mice in Group A received no treatment as the control. The mice in Group B received the treatment of pure PLGA nanofibrous mats $(10 \times 10 \mathrm{~mm}$ in size $)$ on day 12 after HPV + TC-1 injection. The four mice in Group C received PLGA nanofibrous mats with 3:1 polymer-to-drug ratio (PLGA/AG) on day 12 after HPV + TC-1 injection. The wound was closed using 3-0 vicryl sutures post-implantation (Figure 2).

Tissue samples from around the nanofibers were collected from mice receiving AG-loaded mats on days 7, 14, 21, and 28 after operation $(\mathrm{N}=3)$. In vivo drug release was analyzed by HPLC assay. Another nine mice implanted with drugloaded nanofibrous mats were sacrificed on days 1,2, and 3 after operation $(\mathrm{N}=3)$, for histological examination and blood samplings.

\section{Results}

By employing the electrospinning technique, biodegradable nanofibrous mats were successfully prepared. Figure 3 shows 

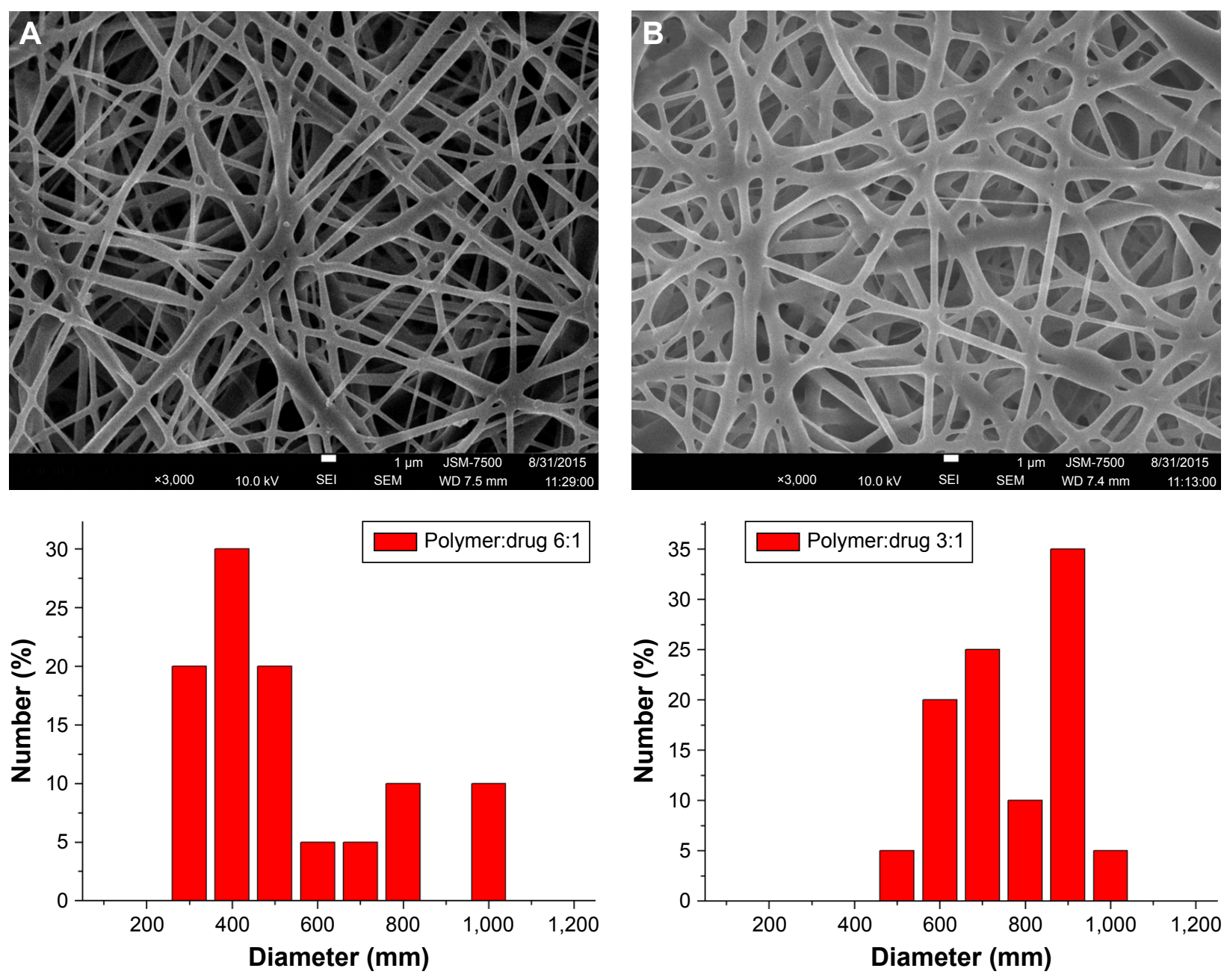

Figure 3 Scanning electron microscope images and fiber diameter distributions of (A) 6:1 and (B) 3:1 polymer-to-drug ratios.

the images and fiber diameter distributions of the spun PLGA/AG nanofibers. The computed fiber diameters were $597.7 \pm 248.1 \mathrm{~nm}$ and $810.6 \pm 133.8 \mathrm{~nm}$, respectively, for the 6:1 and 3:1 polymer-to-drug ratio nanofibers. Nanofiber diameters reduced with the contents of embedded AG. This might be owed to the fact that the polymeric materials in the matrix reduced as the pharmaceutical concentration was raised. Generally, a solution that possesses lower polymer contents is less viscous and, therefore, easier to be stretched when subjected to the same electric force. Fiber diameters reduced accordingly.

The results of the water contact angle estimation in Figure 4 suggest that the contact angles decreased with the increase of AG content in the mat $\left(127.77^{\circ}, 124.22^{\circ}\right.$, and $119.46^{\circ}$, respectively, for the pure PLGA nanofibers, and 6:1 and 3:1 polymer-to-drug ratio nanofibers). Since AG is a poor water-soluble drug, its appearance slightly increases the hydrophilicity of electrospun nanofibers. On the other hand, the tensile test results in Figure 5 show that the ultimate tensile strengths and elongations at the break of spun nanofibers reduced with the increase of contents of embedded AG. This might be due to the fact that the addition of AG reduced the polymer content in the nanofibrous mats. Measured tensile properties were thus compromised accordingly.

An FTIR spectroscopy assay confirmed the AG in the spun nanofibrous membranes. The estimated result in Figure 6 suggested that the absorbance at $3,325 \mathrm{~cm}^{-1}$ was extremely enhanced by the $\mathrm{O}-\mathrm{H}$ bonds of $\mathrm{AG}$. The new peak at $1,688 \mathrm{~cm}^{-1}$ could be attributed to the typical skeletal vibration of the lactone ring $\left(\mathrm{C}_{6} \mathrm{H}_{12} \mathrm{O}_{6}\right) \cdot{ }^{19}$ Furthermore, the $\mathrm{C}=\mathrm{O}$ stretching peaks at $1,710 \mathrm{~cm}^{-1}$ intensively grew relative to the peak of pure PLGA nanofibers. Based on the FTIR spectra, we successfully loaded AG onto the electrospun nanofibers.

\section{In vitro and in vivo liberation behavior of $A G$}

The liberation patterns of $A G$ from the nanofiber are shown in Figure 7. AG showed a steady and nearly first-order drug release pattern for 30 days, after which minor peak releases were observed. The characteristic of daily liberation was 
A
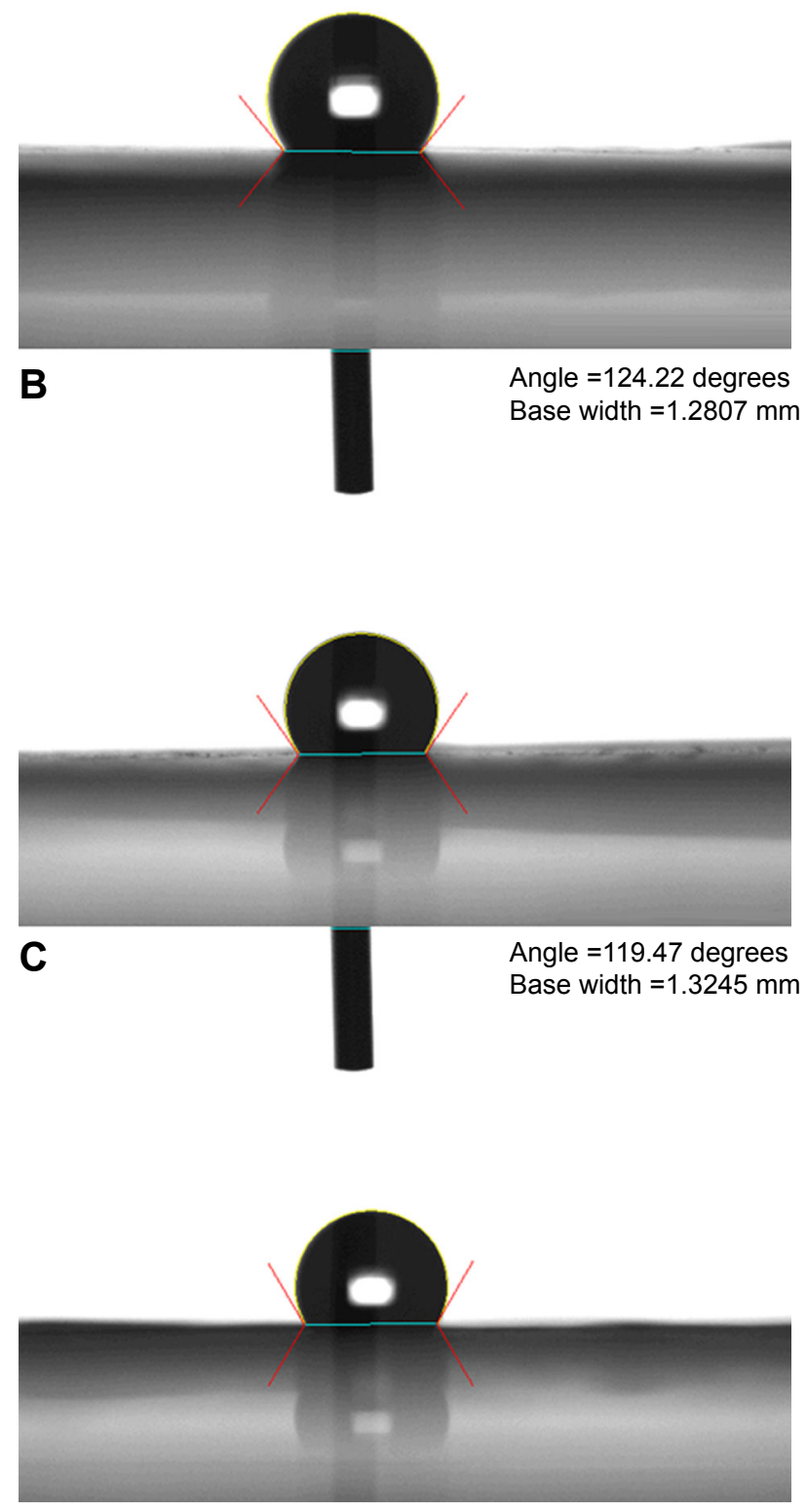

Figure 4 Water contact angles of $(\mathbf{A})$ pure poly[(D,L)-lactide-co-glycolide] nanofibers, and (B) 6:I and (C) 3:I polymer-to-drug ratio nanofibers.

comparable with the two drug-eluting nanofibers of different AG loadings, with a tiny error for the two curves, demonstrating that the AG was evenly embedded in the spun nanofibers. While the 6:1 polymer-to-drug ratio mats released nearly $90 \%$ of the total dosage in 40 days, the $3: 1$ ones only eluted $<80 \%$ of the total drug loaded. This can be explained by the fact that the daily releases of nanofibers with different polymer-to-drug ratios were comparable. The 3:1 polymer-to-drug ratio nanofibers had a greater drug loading,

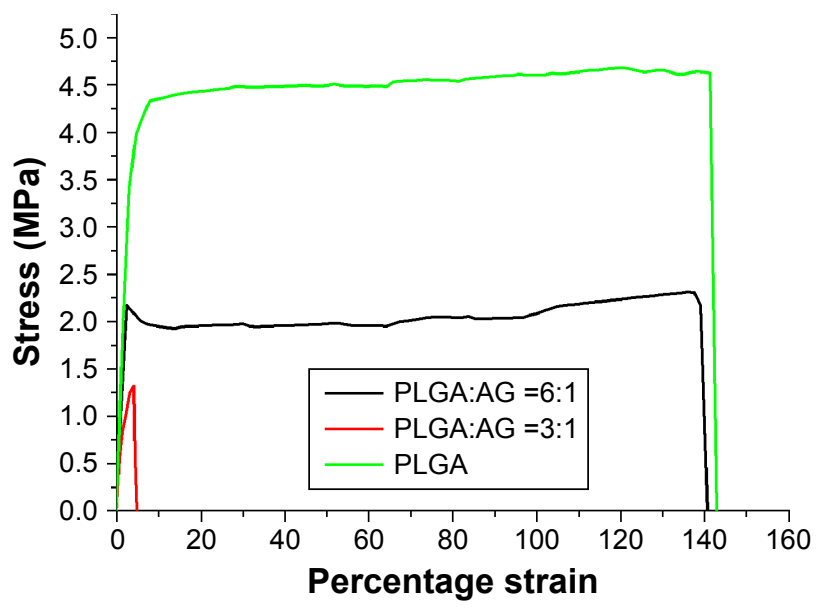

Figure 5 Tensile properties of electrospun drug-eluting nanofibers. Abbreviations: AG, andrographolide; PLGA, poly[(D,L)-lactide-co-glycolide].

based on the comparable daily release, thus exhibited a slower accumulated release. All drug-eluting nanofibers liberated effective levels of $A G$ for $>40$ days.

The in vivo drug release behavior was also investigated. The measured curves in Figure 8 suggest that the drugloaded nanofibers released high concentrations of AG for up to 3 weeks, after which the drug concentration dropped to $1,000 \mu \mathrm{g} / \mathrm{mL}$ at the fourth week. Relatively, the drug level in the plasma showed a concentration significantly lower than the concentration at the target site.

\section{Efficacy of drug-loaded nanofibrous membranes}

Tumor size

Figure 9 displays the gross tumor conditions for the mice in Groups A (control) and C (implanted with drug-eluting

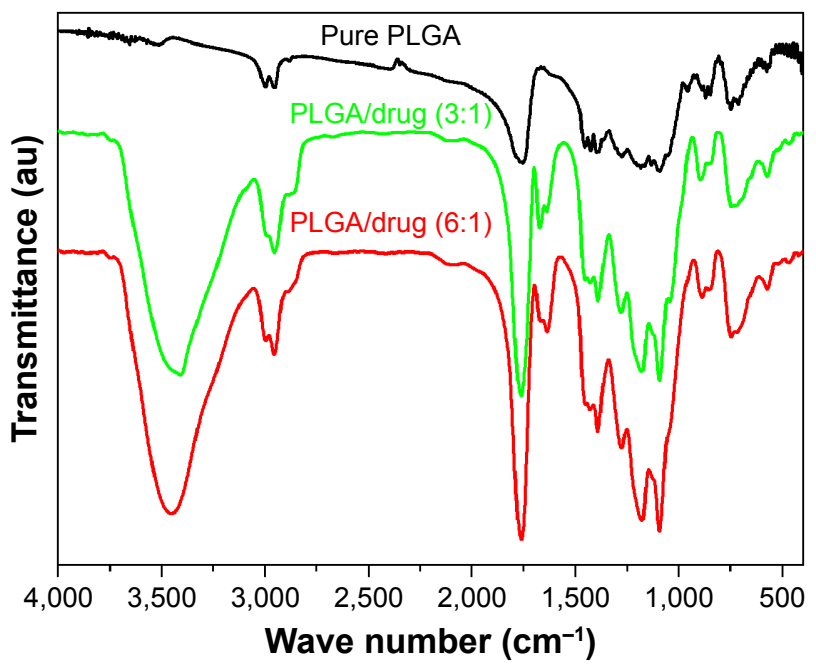

Figure 6 FTIR spectra of pure PLGA and andrographolide-loaded PLGA nanofibers. Abbreviations: FTIR, Fourier transform infrared; PLGA, poly[(D,L)-lactide-coglycolide]. 

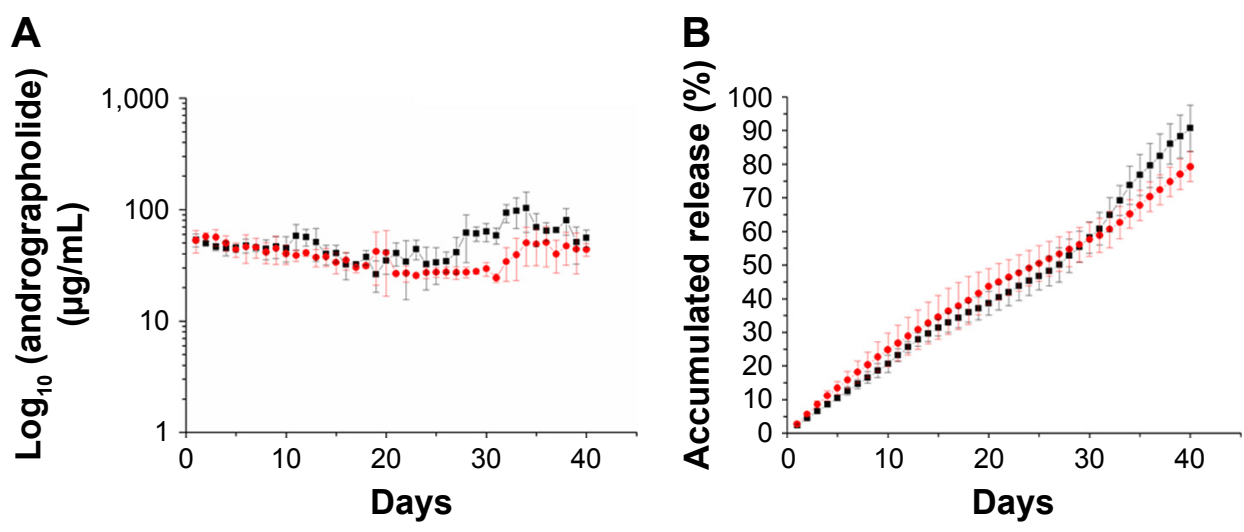

-- Polymer:drug 6:1 $\quad-\bullet-$ Polymer:drug 3:1

Figure 7 Daily $(\mathbf{A})$ and accumulated release $(\mathbf{B})$ of drug-eluting nanofibers in vitro.

nanofibers) at different weeks, while Figure 10 shows the measured size variation with time. While the tumors in Group A (control) and Group B (treated with pure PLGA mats with no drug loading) grew with time, the tumor size in Group C decreased with time until the twenty-fifth day, after which the tumors recurred.

\section{Histological analysis}

The histological images at every time-point (at post-operative days 1, 2, and 3) are shown in Figure 11. No inflammation, increased leukocytes, or tissue necrosis was observed in the histological examination in all groups.

\section{Discussion}

Advanced cervical carcinoma is a primary disease associated with a high mortality rate. Most cervical carcinomas result from infection with HPVs. Despite the use of prophylactic vaccines, the treatment of cervical carcinoma is still

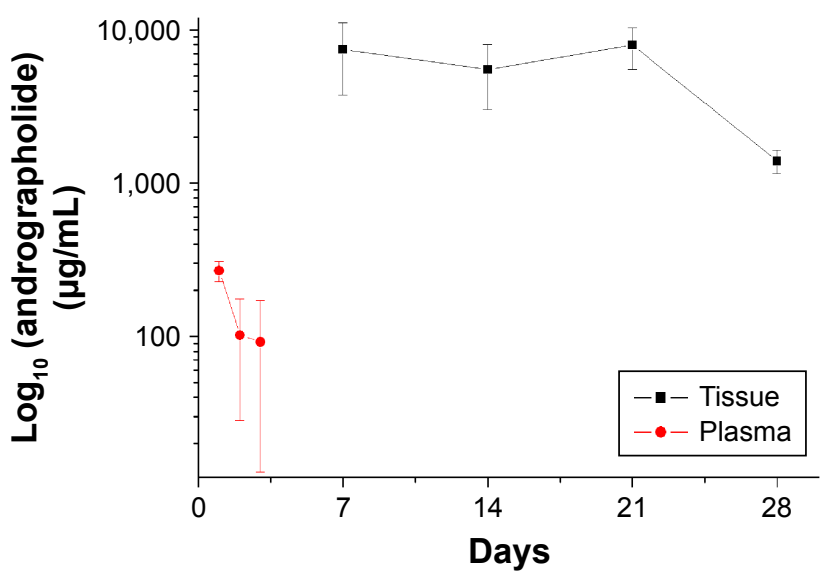

Figure 8 In vivo drug concentrations of andrographolide at the targeted tissue and in the plasma. unsatisfactory. ${ }^{20}$ The excessive number of deaths associated with this cancer could be attributable to the fact that the majority of cervical carcinomas are only identified in advanced stages. Metastatic cervical cancers possess a 5-year survival rate of $\sim 50 \%$. Advanced-stage cervical cancers are treated by different regimes, including chemotherapeutic agents, radiotherapy, and immunotherapy, among others. Nevertheless, these treatments can fail due to drug resistance or rapid tumor progression. In addition, the anticancer therapies of anticarcinoma drugs may induce side effects, which restrict the use of high dosages. Therefore, improved diagnosis and treatment of cervical cancers are highly desired in order to improve the survival rate of the affected individuals.

The cervical epithelium is generally a normal barrier for the prevention of HPV infection. ${ }^{21}$ However, the current cervical screening method, which includes Pap smears and biopsies, may damage the cervical epithelium. In addition, the protocols used for treating CIN, such as laser and cervical conization, also destroy the epithelia. As a result, it would be necessary to use local agents to protect the barrier from iatrogenic cervical lesion to prevent further HPV infections.

In recent years, nanotechnology has provided different ways to enhance the management of carcinomas; it appears to be a promising methodology for developing cancer treatments. Nanoparticles are able to accumulate at the tumor site via a passive targeting strategy, thereby reducing the side effects of pharmaceuticals and enhancing treatment effectiveness. ${ }^{22}$ Cancer-targeting delivery has also been investigated to improve the accumulation of anti-tumor pharmaceuticals at tumor sites. In cervical cancer, nanotechnology has been increasingly studied for enhancing early diagnosis and improving vaccine and treatment efficacy. ${ }^{23}$ Roy et $\mathrm{al}^{24}$ pointed out that the use of nanoparticles improves 
A0

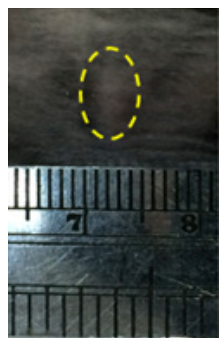

B0

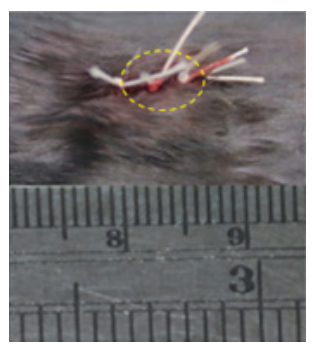

Co

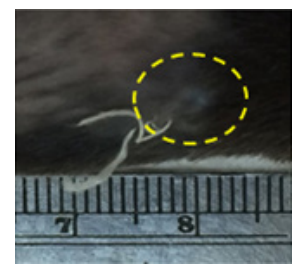

A7

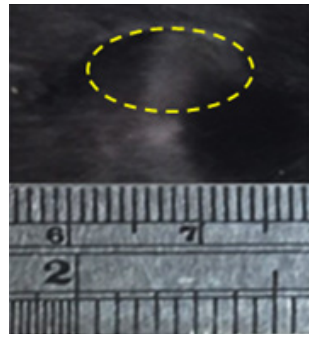

B7

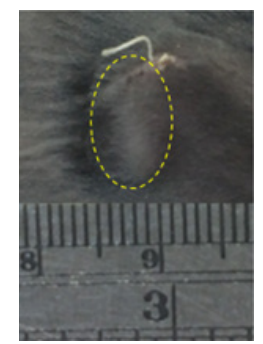

C7

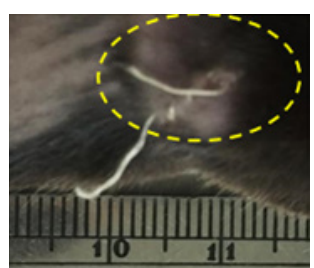

A14

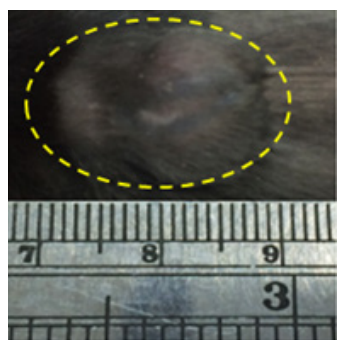

B14

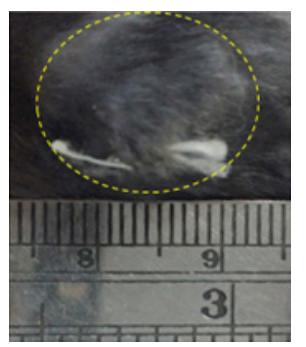

C14

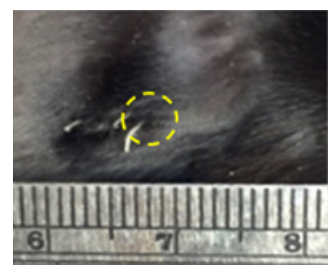

A21

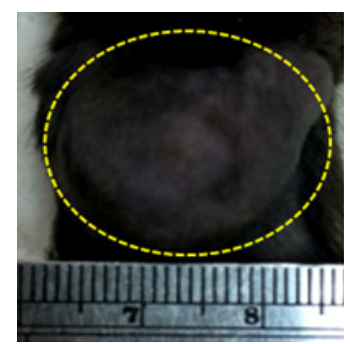

B21

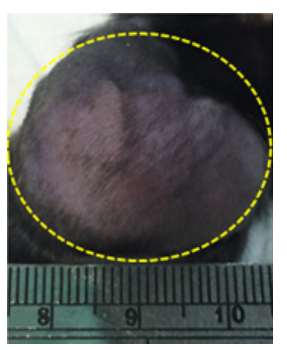

C21

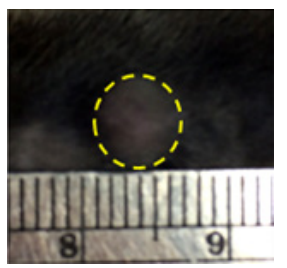

A28

Sacrificed

B28

Sacrificed

C28

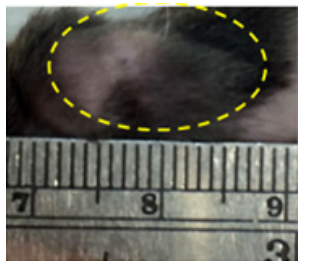

Figure 9 Images of the tumors on the backs of the mice.

Notes: A represents as group A; B represents as group B; C represents as group C. 0 represents as day 0; 7 represents as day $7 ; 14$ represents as day I4; 2 I represents as day $21 ; 28$ represents as day 28 .

the effectiveness of anticancer AG in MCF-7 cells and mice with Ehrlich ascites carcinoma. Parveen et $\mathrm{al}^{25}$ formulated lipid AG nanoparticles and examined the in vitro and in vivo efficacy. They found that the anticancer activity in Balb/c

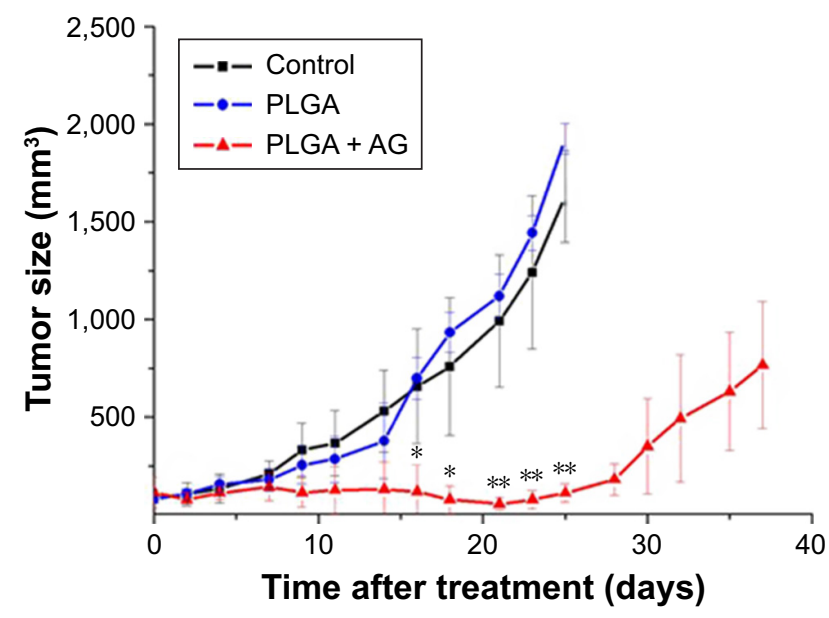

Figure 10 Variation of tumor sizes with time.

Notes: $* P<0.05$ and $* * p<0.01$.

Abbreviations: AG, andrographolide; PLGA, poly[(D,L)-lactide-co-glycolide]. mice showed better outcomes with AG-loaded solid lipid nanoparticles compared with the $\mathrm{AG}$ alone. Zhang et $\mathrm{al}^{26}$ proposed AG-embedded PLGA/PEG/PLGA micelles to enhance the drug's bioactivity and anticancer efficacy.

This study developed biodegradable PLGA nanofibrous membranes for the sustained transport of AG to the target tumor site for the treatment of cervical cancers. Owing to the high surface area-to-volume ratio, nanofibers provide a useful pathway for delivery of water-insoluble or poor water-soluble drugs. Electrospun drug-embedded nanofibers not only possess one dimension at the microscopic scale but another dimension in the macroscopic form. This distinct feature endows the drug-loaded nanofibers with the advantage in modifying the biopharmaceutic and/or pharmacokinetic characteristics of the drug molecule for desired clinical outcomes and the merits of traditional solid dosage forms such as simple processing, good drug stability, ease of packaging and shipping. ${ }^{27}$

Different biomaterials have been used for the transport of pharmaceuticals, genes, and peptides. Among these materials, PLGA is a biodegradable polymer that has received the approval of pharmaceutical agents as a therapeutic carrier 

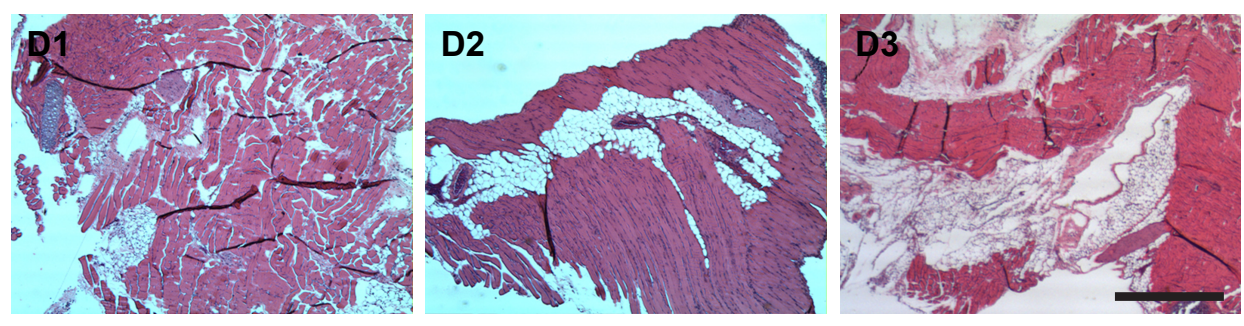

Figure I I Histological analysis images at days I-3 (DI-D3). Notes: Scale bar I mm.

owing to its good biocompatibility and biodegradability. ${ }^{28,29}$ Local drug therapy with pharmaceuticals incorporated into implants or administered by local injection or implantation provides the advantages of delivering high drug levels to the target site while minimizing the systemic drug concentrations and the associated side effects. The experimental results in this work demonstrated that the AG-eluting nanofibrous membranes exhibit a steady and nearly first-order in vitro drug release pattern for 30 days, after which minor peak releases were observed. Meanwhile, the drug levels in the blood remained much lower. Of clinical importance, the animal study showed that the biodegradable drug-embedded nanofibers provide a sustained liberation of AG for 4 weeks in vivo. This extended liberation of therapeutic levels of AG provides specific benefits in terms of managing cervical cancers as well as preventing their recurrence.

The animal test results showed that while the tumor size for rats in the control and in the group receiving pure PLGA mats grew with time, the tumor size in rats implanted with AGeluting mats decreased over time until day 25 . Thereafter, the tumor recurred. This might be attributable to the fact that the drug-loaded nanofibers released high concentrations of AG for up to 3 weeks, after which the drug concentration gradually dropped to $1,000 \mu \mathrm{g} / \mathrm{mL}$ at the fourth week. Tumor cells thus regenerated with the decrease of drug concentration.

\section{Limitations}

In spite of the proven efficacy of the AG-embedded nanofibers, there still exist limitations in this study. The first limitation is the relatively small number of animals enrolled in this study. The second limitation is the relevance of our findings to humans with cervical cancer, which remains to be consolidated and requires further exploration.

\section{Conclusion}

In this study, we manufactured biodegradable AG-eluting nanofibrous mats and evaluated their efficacy in treating cervical cancers, employing PLGA as the carrying matrix.
The in vitro AG release was evaluated by HPLC. A cervical cancer mouse model was created and employed for the in vivo efficacy assessment of drug-eluting nanofibers. The experimental results demonstrated that all membranes liberated effective concentrations of $\mathrm{AG}$ for $>4$ weeks after surgery while the systemic drug concentration in the blood remained low. Histological analysis showed no obvious tissue inflammation. Therefore, we conclude that nanofibers with sustainable AG releases exhibit their potential in the treatment of cervical cancer.

\section{Acknowledgments}

This work was supported financially by the Ministry of Science and Technology, Taiwan (contract no. 107-2221-E182-017) and the Chang Gung Memorial Hospital-Linkou (contract no. CMRPD2H0031).

\section{Disclosure}

The authors report no conflicts of interest in this work.

\section{References}

1. Peralta-Zaragoza O, Bermúdez-Morales VH, Pérez-Plasencia C, SalazarLeón J, Gómez-Cerón C, Madrid-Marina V. Targeted treatments for cervical cancer: a review. Onco Targets Ther. 2012;5:315-328.

2. Elit L, Fyles AW, Devries MC, Oliver TK, Fung-Kee-Fung M; Gynecology Cancer Disease Site Group. Follow-up for women after treatment for cervical cancer: a systematic review. Gynecol Oncol. 2009;114(3): $528-535$.

3. Doorbar J, Egawa N, Griffin H, Kranjec C, Murakami I. Human papillomavirus molecular biology and disease association. Rev Med Virol. 2015;25(Suppl 1):2-23.

4. Schiffman M, Solomon D. Clinical practice. Cervical-cancer screening with human papillomavirus and cytologic cotesting. $N$ Engl $J$ Med. 2013;369(24):2324-2331.

5. Gadducci A, Tana R, Cosio S, Cionini L. Treatment options in recurrent cervical cancer (Review). Oncol Lett. 2010;1(1):3-11.

6. Kumar N. Cervical cancer; a nightmare for womanhood: review of recent advances. Women's Health \& Gynecology. 2016;2(2):017(1-9).

7. Zhang Z, Wu Y, Kuang G, et al. Pt(iv) prodrug-backboned micelle and DCA loaded nanofibers for enhanced local cancer treatment. J Mater Chem B. 2017;5(11):2115-2125.

8. Zhang Z, Liu S, Qi Y, et al. Time-programmed DCA and oxaliplatin release by multilayered nanofiber mats in prevention of local cancer recurrence following surgery. J Control Release. 2016; 235:125-133. 
9. Ma Y, Wang X, Zong S, et al. Local, combination chemotherapy in prevention of cervical cancer recurrence after surgery by using nanofibers co-loaded with cisplatin and curcumin. RSC Adv. 2015;5(129): 106325-106332.

10. Jada SR, Subur GS, Matthews C, et al. Semisynthesis and in vitro anticancer activities of andrographolide analogues. Phytochemistry. 2007;68(6):904-912.

11. Song Y, Xin Z, Wan Y, Li J, Ye B, Xue X. Synthesis and anticancer activity of some novel indolo[3,2-b]andrographolide derivatives as apoptosis-inducing agents. Eur J Med Chem. 2015;90:695-706.

12. Lim JC, Jeyaraj EJ, Sagineedu SR, Wong WS, Stanslas J. SRS06, a new semisynthetic andrographolide derivative with improved anticancer potency and selectivity, inhibits nuclear factor- $\kappa \mathrm{B}$ nuclear binding in the A549 non-small cell lung cancer cell line. Pharmacology. 2015;95(1-2): 70-77.

13. Wang J, Tan XF, Nguyen VS, Yang P, Zhou J. A quantitative chemical proteomics approach to profile the specific cellular targets of andrographolide, a promising anticancer agent that suppresses tumor metastasis Mol Cell Proteomics. 2014;13(3):876-886.

14. Gunn EJ, Williams JT, Huynh DT, et al. The natural products parthenolide and andrographolide exhibit anti-cancer stem cell activity in multiple myeloma. Leuk Lymphoma. 2011;52(6):1085-1097.

15. Menon V, Bhat B. Anticancer activity of andrographolide semisynthetic derivatives. Nat Prod Commun. 2010;5(5):717-720.

16. Xia YF, Ye BQ, Li YD, et al. Andrographolide attenuates inflammation by inhibition of NF-kappa B activation through covalent modification of reduced cysteine 62 of p50. J Immunol. 2004;173(6):4207-4217.

17. Kao CW, Lee D, Wu MH, Chen JK, He HL, Liu SJ. Lidocaine/ketorolacloaded biodegradable nanofibrous anti-adhesive membranes that offer sustained pain relief for surgical wounds. Int J Nanomedicine. 2017; 12:5893-5901.

18. Lin CC, Tsai CC, Lee JM, et al. The efficacy of a novel vaccine approach using tumor cells that ectopically express a codon-optimized murine GM-CSF in a murine tumor model. Vaccine. 2016;34(1):134-141.
19. Singh PK, Hasan T, Prasad O, Sinha L, Raj K, Misra N. FT-IR spectra and vibrational spectroscopy of Andrographolide. Spectroscopy. 2006; 20(5-6):275-283.

20. Mckee SJ, Bergot AS, Leggatt GR. Recent progress in vaccination against human papillomavirus-mediated cervical cancer. Rev Med Virol. 2015;25(Suppl 1):54-71.

21. Chen J. Signaling pathways in HPV-associated cancers and therapeutic implications. Rev Med Virol. 2015;25(Suppl 1):24-53.

22. Wong IY, Bhatia SN, Toner M. Nanotechnology: emerging tools for biology and medicine. Genes Dev. 2013;27(22):2397-2408.

23. Chen J, Gu W, Yang L, et al. Nanotechnology in the management of cervical cancer. Rev Med Virol. 2015;25(Suppl 1):72-83.

24. Roy P, das S, Mondal A, Chatterji U, Mukherjee A. Nanoparticle engineering enhances anticancer efficacy of andrographolide in MCF-7 cells and mice bearing EAC. Curr Pharm Biotechnol. 2012;13(15): 2669-2681.

25. Zhang J, Li Y, Gao W, Repka MA, Wang Y, Chen M. Andrographolideloaded PLGA-PEG-PLGA micelles to improve its bioavailability and anticancer efficacy. Expert Opin Drug Deliv. 2014;11(9):1367-1380.

26. Parveen R, Ahmad FJ, Iqbal Z, Samim M, Ahmad S. Solid lipid nanoparticles of anticancer drug andrographolide: formulation, in vitro and in vivo studies. Drug Dev Ind Pharm. 2014;40(9):1206-1212.

27. Yu DG, Zhu LM, White K, Branford-White C. Electrospun nanofiberbased drug delivery systems. Health. 2009;1(2):67-75.

28. Makadia HK, Siegel SJ. Poly lactic-co-glycolic acid (PLGA) as biodegradable controlled drug delivery carrier. Polymers. 2011;3(3): 1377-1397.

29. Gentile P, Chiono V, Carmagnola I, Hatton PV. An overview of poly(lactic-co-glycolic) acid (PLGA)-based biomaterials for bone tissue engineering. Int J Mol Sci. 2014;15(3):3640-3659.
International Journal of Nanomedicine

\section{Publish your work in this journal}

The International Journal of Nanomedicine is an international, peerreviewed journal focusing on the application of nanotechnology in diagnostics, therapeutics, and drug delivery systems throughout the biomedical field. This journal is indexed on PubMed Central, MedLine, CAS, SciSearch $®$, Current Contents $\AA /$ Clinical Medicine,

\section{Dovepress}

Journal Citation Reports/Science Edition, EMBase, Scopus and the Elsevier Bibliographic databases. The manuscript management system is completely online and includes a very quick and fair peer-review system, which is all easy to use. Visit http://www.dovepress.com/ testimonials.php to read real quotes from published authors. 